DOI 10.4171/JEMS/247

Stephen S.-T. Yau

\title{
Rigidity of CR morphisms between compact strongly pseudoconvex CR manifolds
}

\author{
This paper is dedicated to Professor Heisuke Hironaka \\ on the occasion of his 77 th birthday
}

Received August 29, 2007 and in revised form October 4, 2008

\begin{abstract}
Let $X_{1}$ and $X_{2}$ be two compact strongly pseudoconvex CR manifolds of dimension $2 n-1 \geq 5$ which bound complex varieties $V_{1}$ and $V_{2}$ with only isolated normal singularities in $\mathbb{C}^{N_{1}}$ and $\mathbb{C}^{N_{2}}$ respectively. Let $S_{1}$ and $S_{2}$ be the singular sets of $V_{1}$ and $V_{2}$ respectively and assume $S_{2}$ is non-empty. If $2 n-N_{2}-1 \geq 1$ and the cardinality of $S_{1}$ is less than twice that of $S_{2}$, then we prove that any non-constant CR morphism from $X_{1}$ to $X_{2}$ is necessarily a CR biholomorphism. On the other hand, let $X$ be a compact strongly pseudoconvex CR manifold of dimension 3 which bounds a complex variety $V$ with only isolated normal non-quotient singularities. Assume that the singular set of $V$ is non-empty. Then we prove that any non-constant CR morphism from $X$ to $X$ is necessarily a CR biholomorphism.
\end{abstract}

Keywords. Strongly pseudoconvex CR manifold, rigidity of CR morphism, geometric genus of compact embeddable CR manifold

\section{Introduction}

CR manifolds are abstract models of boundaries of complex manifolds. Strongly pseudoconvex CR manifolds have rich geometric and analytic structures. The harmonic theory for the $\bar{\partial}_{b}$ complex on compact strongly pseudoconvex CR manifolds was developed by Kohn $[\mathrm{Ko}]$. Using this theory, Boutet de Monvel $[\mathrm{BM}]$ proved that if $X$ is a compact strongly pseudoconvex CR manifold of dimension $2 n-1, n \geq 3$, then there exist $C^{\infty}$ functions $f_{1}, \ldots, f_{N}$ on $X$ such that each $\bar{\partial}_{b} f_{j}=0$ and $f=\left(f_{1}, \ldots, f_{N}\right)$ defines an embedding of $X$ in $\mathbb{C}^{N}$. Thus, any compact strongly pseudoconvex CR manifold of dimension $\geq 5$ can be $\mathrm{CR}$ embedded in some complex Euclidean space. On the other hand, 3-dimensional strongly pseudoconvex compact orientable CR-manifolds are not necessarily embeddable. Throughout this paper, our strongly pseudoconvex CR manifolds are always assumed to be compact orientable and embeddable. By the classical theorem of Harvey and Lawson [Ha-La1], [Ha-La2], a strongly pseudoconvex complex embeddable

S. S.-T. Yau: Institute of Mathematics, East China Normal University, Shanghai, China; current address: Department of Mathematics, Statistics and Computer Science (M/C 249), Rm 322, SEO, University of Illinois at Chicago, 851 S. Morgan Street, Chicago, IL 60607-7045, U.S.A.; e-mail: yau@uic.edu

Mathematics Subject Classification (2010): Primary 32V99, 32S20; Secondary 14E20 
$\mathrm{CR}$ manifold can be embedded in some $\mathbb{C}^{N}$ and bounds a Stein variety with at most isolated normal singularities.

In the remarkable paper [Pi], Pinchuk considered proper holomorphic maps of strongly pseudoconvex domains in $\mathbb{C}^{n}$ that are smooth up to the boundary. (It was shown later in [Be-Ca] and [Di-Fo] that the smoothness assumption is satisfied automatically.) Pinchuk proved that such maps are locally biholomorphic. Moreover, he showed that a proper holomorphic self-map of a strongly pseudoconvex domain is biholomorphic. Since CR maps between strongly pseudoconvex boundaries extend to holomorphic maps of the corresponding domains, Pinchuk's results may be interpreted as rigidity statements about CR maps of strongly pseudoconvex compact hypersurfaces in $\mathbb{C}^{n}$.

In the present paper, we investigate rigidity properties of CR maps of general embeddable strongly pseudoconvex compact CR manifolds. Our starting point is the following result.

Proposition 1.1. Let $X_{1}$ and $X_{2}$ be two compact strongly pseudoconvex CR manifolds of dimension $2 n-1 \geq 3$ which bound complex varieties $V_{1}$ and $V_{2}$ in $\mathbb{C}^{N_{1}}$ and $\mathbb{C}^{N_{2}}$ respectively. Suppose the singular set $S_{i}$ of $V_{i}, i=1,2$, is either an empty set or a set consisting of only isolated normal singularities. If $\Phi: X_{1} \rightarrow X_{2}$ is a non-constant CR morphism, then $\Phi$ is surjective and $\Phi$ can be extended to a proper surjective holomorphic map from $V_{1}$ to $V_{2}$ such that $\Phi\left(S_{1}\right) \subseteq S_{2}, \Phi^{-1}\left(X_{2}\right)=X_{1}$ and $\Phi: V_{1}-\Phi^{-1}\left(S_{2}\right) \rightarrow V_{2}-S_{2}$ is a covering map. Moreover, if $S_{2}$ does not have quotient singularity, then $\Phi^{-1}\left(S_{2}\right)=S_{1}$.

As a corollary of Proposition 1.1, we have the following super-rigidity results for CR morphisms between strongly pseudoconvex manifolds.

Corollary 1.1. Let $X_{1}$ be a compact strongly pseudoconvex CR manifold of dimension $2 n-1 \geq 3$ which bounds a complex variety $V_{1}$ in $\mathbb{C}^{N_{1}}$ with isolated normal singularities. Let $X_{2}$ be a compact strongly pseudoconvex $C R$ manifold of dimension $2 n-1$ which bounds a complex submanifold $V_{2}$ in $\mathbb{C}^{N_{2}}$. Then there is no non-constant CR morphism from $X_{1}$ to $X_{2}$.

For any compact strongly pseudoconvex embeddable CR manifold $X$, one can define a notion of geometric genus $p_{g}(X)$ which is a non-negative integer and is invariant under CR biholomorphism (see Definition 2.4, Proposition 2.2 and Remark 2.1 below).

Proposition 1.2. Let $X_{1}$ be a compact strongly pseudoconvex $C R$ manifold of dimension $2 n-1 \geq 3$ which bounds a complex submanifold $V_{1}$ in $\mathbb{C}^{N_{1}}$. Let $X_{2}$ be a compact strongly pseudoconvex $C R$ manifold of dimension $2 n-1$ with either (i) geometric genus $p_{g}\left(X_{2}\right)>0$ or (ii) $p_{g}\left(X_{2}\right)=0$ and $X_{2}$ bounds a complex variety $V_{2}$ in $\mathbb{C}^{N_{2}}$ with a non-quotient singularity. Then there is no non-constant CR morphism from $X_{1}$ to $X_{2}$.

Remark 1.1. Proposition 1.2 is false if $p_{g}\left(X_{2}\right)=0$ and $V_{2}$ has only quotient singularities in the interior, as we can see from the following example.

Example 1.1. Let $B=\left\{(x, y) \in \mathbb{C}^{2}:|x|^{2}+|y|^{2}<1\right\}$ and $S=\partial B$. In the notation of Proposition 1.2, let $X_{1}=S$ be the standard sphere and $V_{1}=B$. Let $\sigma: B \rightarrow B$ 
be the map given by $\sigma(x, y)=(-x,-y)$. Let $V_{2}$ be the quotient of $V_{1}$ by the cyclic group of order 2 generated by $\sigma$. Then $V_{2}$ is a strongly pseudoconvex variety with $A_{1}$ singularity $\left\{\left(z_{1}, z_{2}, z_{3}\right) \in \mathbb{C}^{3}: z_{1} z_{2}=z_{3}^{2}\right\}$. The quotient map $\Phi: V_{1} \rightarrow V_{2}$ is given by $\left(z_{1}, z_{2}, z_{3}\right)=\left(x^{2}, y^{2}, x y\right)$. Clearly $\Phi$ sends $X_{1}$ surjectively onto $X_{2}=\partial V_{2}$ and $\Phi$ is a non-constant CR morphism.

Proposition 1.3. Let $X_{1}$ and $X_{2}$ be two compact strongly pseudoconvex embeddable CR manifolds of dimension $2 n-1 \geq 3$. If there is a non-constant $C R$ morphism from $X_{1}$ to $X_{2}$, then $p_{g}\left(X_{1}\right) \geq p_{g}\left(X_{2}\right)$.

Corollary 1.2. Let $X_{1}, X_{2}$ be two compact strongly pseudoconvex embeddable CR manifolds of dimension $2 n-1 \geq 3$. If $p_{g}\left(X_{1}\right)<p_{g}\left(X_{2}\right)$, then there is no non-constant $C R$ morphism from $X_{1}$ to $X_{2}$.

The following theorem says that if the codimension of $X_{2}$ is small and $\operatorname{dim} X_{2} \geq 5$, then there is no non-constant CR morphism from $X_{1}$ to $X_{2}$ except CR biholomorphic maps. This rigidity phenomenon does not require any curvature assumption on $X_{1}$ or $X_{2}$.

Theorem 1.1. Let $X_{1}$ and $X_{2}$ be two compact strongly pseudoconvex CR manifolds of dimension $2 n-1 \geq 5$ which bound complex varieties $V_{1}$ and $V_{2}$ with only isolated normal singularities in $\mathbb{C}^{\bar{N}_{1}}$ and $\mathbb{C}^{N_{2}}$ respectively. Let $S_{1}$ and $S_{2}$ be the singular sets of $V_{1}$ and $V_{2}$ respectively and suppose $S_{2}$ is non-empty. If $2 n-N_{2}-1 \geq 1$ and $\left|S_{1}\right| \leq 2\left|S_{2}\right|-1$, then every non-constant CR morphism from $X_{1}$ to $X_{2}$ is necessarily a CR biholomorphism.

Let $X$ be a compact strongly pseudoconvex CR manifold of dimension $2 n-1$ embeddable in $\mathbb{C}^{N}$. We would like to know whether a non-constant CR morphism from $X$ to $X$ is necessarily a CR biholomorphism. If $\operatorname{dim} X \geq 5$, then Theorem 1.1 above gives us an affirmative answer so long as $2 n-N-1 \geq 1$. If $\operatorname{dim} X=3$, the situation is more complex. In Theorem 1.2, a stronger result is proved without the assumption on the codimension of $X$ in $\mathbb{C}^{N}$.

Theorem 1.2. Let $X$ be a compact strongly pseudoconvex CR manifold of dimension 3 in $\mathbb{C}^{N}$ which bounds a complex variety $V$ with only isolated normal non-quotient singularities. Assume that the singular set of $V$ is non-empty. Then every non-constant $C R$ morphism from $X$ to $X$ is necessarily a CR biholomorphism.

In the course of proving Theorem 1.2, we have proved the following theorem, which is of independent interest.

Theorem 1.3. Let $V$ be a 2-dimensional Stein space with only isolated normal singularities. Let $\Phi: V \rightarrow V$ be a holomorphic map from $V$ to $V$ such that $\Phi^{-1}(S)=S$, where $S$ is the non-empty singular set of $V$. If $\Phi: V-S \rightarrow V-S$ is a covering map, then $\Phi$ is a biholomorphism.

The proof of Theorem 1.2 does not work if $p_{g}(X)=0$ and $V$ contains a quotient singularity. For such rational compact CR manifolds, Theorem 1.2 may still be true, and we make the following rigidity conjecture. 
Conjecture 1.1. Let $X$ be a compact strongly pseudoconvex embeddable CR manifold of dimension at least 3. Then every non-constant CR morphism from $X$ to itself must be a CR biholomorphism.

The following example shows that strong pseudoconvexity plays an important role in the above theory.

Example 1.2. Let $X_{1}=\left\{(x, y, z) \in \mathbb{C}^{3}: x y=z^{2}, a|x|^{4}+|y|^{4}+|z|^{4}=\epsilon_{0}\right\}$ and $X_{2}=\left\{(x, y, z) \in \mathbb{C}^{3}: x y=z^{2}, a|x|^{2}+|y|^{2}+|z|^{2}=\epsilon_{0}\right\}$ where $a$ is a positive real number. Let $\psi: X_{1} \rightarrow X_{2}$ be given by $\psi(x, y, z)=\left(x^{2}, y^{2}, z^{2}\right)$. Then $\psi$ is a surjective CR morphism from $X_{1}$ to $X_{2}$, but $\psi$ is not a CR biholomorphism. Note that $X_{2}$ is strongly pseudoconvex, but $X_{1}$ is only weakly pseudoconvex.

As another evidence to support our Conjecture 1.1, we have the following result which follows from Pinchuk's argument in [Pi].

Proposition 1.4. Let $X$ be a compact strongly pseudoconvex $C R$ manifold of dimension $2 n-1 \geq 3$. If $X$ bounds a complex submanifold $V$ in $\mathbb{C}^{N}$, then every non-constant $C R$ morphism from $X$ to $X$ is a CR biholomorphism.

In Section 2, we shall recall some basic notions of CR manifolds. Propositions 1.1 to 1.4 and Theorems 1.1 to 1.3 are proved in Section 3.

\section{Preliminaries on CR manifolds}

Definition 2.1. Let $X$ be a connected orientable manifold of real dimension $2 n-1$. A $C R$ structure on $X$ is an $(n-1)$-dimensional subbundle $S$ of the complexified tangent bundle $\mathbb{C} T X$ such that

(1) $S \cap \bar{S}=\{0\}$.

(2) If $L, L^{\prime}$ are local sections of $S$, then so is $\left[L, L^{\prime}\right]$.

A manifold with a CR structure is called a $C R$ manifold. There is a unique subbundle $\mathcal{H}$ of the tangent bundle $T(X)$ such that $\mathbb{C H}=S \oplus \bar{S}$

Furthermore, there is a unique homomorphism $J: \mathcal{H} \rightarrow \mathcal{H}$ such that $J^{2}=-1$ and $S=\{v-i J v: v \in \mathcal{H}\}$. The pair $(\mathcal{H}, J)$ is called the real expression of the CR structure.

Definition 2.2. Let $L_{1}, \ldots, L_{n-1}$ be a local frame of $S$. Then $\bar{L}_{1}, \ldots, \bar{L}_{n-1}$ is a local frame of $\bar{S}$ and one may choose a local section $N$ of $T X$ which is purely imaginary, such that $L_{1}, \ldots, L_{n-1}, \bar{L}_{1}, \ldots, \bar{L}_{n-1}, N$ is a local frame of $\mathbb{C} T X$. The matrix $\left(c_{i j}\right)$ defined by

$$
\left[L_{i}, \bar{L}_{j}\right]=\sum a_{i j}^{k} L_{k}+\sum b_{i j}^{k} \bar{L}_{k}+\sqrt{-1} c_{i j} N
$$

is Hermitian and is called the Levi form of $X$.

Proposition 2.1. The number of non-zero eigenvalues and the absolute value of the signature of the Levi form $\left(c_{i j}\right)$ at each point are independent of the choice of $L_{1}, \ldots, L_{n-1}, N$.

Definition 2.3. The CR manifold $X$ is called strongly pseudoconvex if the Levi form is definite at each point of $X$. 
Theorem 2.1 (Boutet de Monvel [BM]). If $X$ is a compact strongly pseudoconvex $C R$ manifold of dimension $2 n-1$ and $n \geq 3$, then $X$ is CR embeddable in $\mathbb{C}^{N}$.

Although there are non-embeddable compact 3-dimensional CR manifolds, in this paper all CR manifolds are assumed to be embeddable in complex Euclidean space.

Theorem 2.2 (Harvey-Lawson [Ha-La1], [Ha-La2]). For any compact connected embeddable strongly pseudoconvex $C R$ manifold $X$, there is a unique complex variety $V$ in $\mathbb{C}^{N}$ for some $N$ such that the boundary of $V$ is $X$ and $V$ has only normal isolated singularities.

Definition 2.4. Let $X$ be a compact connected strongly pseudoconvex embeddable CR manifold of real dimension $2 n-1$. Let $V$ be the normal subvariety in $\mathbb{C}^{N}$ such that the boundary of $V$ is $X$. Let $\pi: M \rightarrow V$ be a resolution of singularities of $V$. The geometric genus of $X$ denoted by $p_{g}(X)$ is defined to be $\operatorname{dim} H^{n-1}(M, \mathcal{O})$.

Proposition 2.2 ([YYa-Yu] $)$. Let $X$ be a connected compact strongly pseudoconvex $C R$ manifold of real dimension $2 n-1$ and $n \geq 2$. Suppose that $X$ bounds a normal variety $V \subseteq \mathbb{C}^{N}$ with isolated singularities $Y=\left\{q_{1}, \ldots, q_{m}\right\}$. Let $\pi: M \rightarrow V$ be a resolution of singularities of $V$. Then the geometric genus $p_{g}(X):=\operatorname{dim} H^{n-1}(M, \mathcal{O})$ is a $C R$ invariant of $X$. In fact, let $U$ be any strongly pseudoconvex neighborhood of $Y$. Then

$$
p_{g}(X)=\operatorname{dim} H^{0}\left(U-Y, \Omega^{n}\right) / L^{2}\left(U-Y, \Omega^{n}\right)
$$

where $\Omega^{n}$ is the sheaf of germs of holomorphic n-forms and $L^{2}\left(U-Y, \Omega^{n}\right)$ is the space of holomorphic n-forms $\omega$ on $U-Y$ which are $L^{2}$-integrable for some smaller neighborhood $U^{\prime} \subset \subset U$ of $Y$, i.e., $\int_{U^{\prime}-Y} \omega \wedge \bar{\omega}<\infty$.

Definition 2.5. Let $(V, q)$ be a complex analytic variety with isolated normal singularity at $q$ of dimension $n$. The geometric genus $p_{g}(V, q)$ of the singularity $(V, q)$ is defined to be $\operatorname{dim} H^{0}\left(U-\{q\}, \Omega^{n}\right) / L^{2}\left(U-\{q\}, \Omega^{n}\right)$ where $U$ is any strongly pseudoconvex neighborhood of $q$.

Remark 2.1. (a) Let $\pi:(M . A) \rightarrow(V, q)$ be a resolution of singularity. By a theorem of [Ya1], $H^{n-1}(M, \mathcal{O})=\operatorname{dim} H^{0}\left(V-\{q\}, \Omega^{n}\right) / L^{2}\left(V-\{q\}, \Omega^{n}\right)$. It follows easily that $p_{g}(V, q)$ is independent of $U$ in the above definition.

(b) With the notation of Proposition 2.2. we have $p_{g}(X)=\sum_{i=1}^{m} p_{g}\left(V, q_{i}\right)$.

\section{Proofs of Theorems 1.1 to 1.3 and Propositions 1.1 to 1.4}

Proof of Proposition 1.1 Let $\phi_{1}, \ldots, \phi_{N_{2}}$ be the component functions of $\Phi$. Then $\phi_{i}$ as CR holomorphic function on $X_{1}$ can be extended onto a one-sided neighborhood of $X_{1}$ in $V_{1}$. By Andreotti and Grauert ([An-Gr. Théorème 15]), $\phi_{i}$ can be holomorphically extended onto $V_{1}-S_{1}$ where $S_{1}$ is the singular set of $V_{1}$. Since $S_{1}$ is either an empty set or a set consisting of only isolated normal singularities, $\phi_{i}$ can be holomorphically extended onto $V_{1}$. 
We claim that $\Phi\left(V_{1}\right) \subseteq V_{2}$. To see this, let $f_{1}, \ldots, f_{k}$ be the defining equations of $V_{2}$, i.e. $V_{2}=\left\{y \in \mathbb{C}^{N_{2}}: f_{1}(y)=\cdots=f_{k}(y)=0\right\}$. Clearly $\Phi^{*}\left(f_{i}\right)=f_{i} \circ \Phi$ is a holomorphic function on $V_{1}$ which vanishes on $X_{1}$ for $1 \leq i \leq k$. Since $X_{1}$ is of real codimension one in $V_{1}, \Phi^{*}\left(f_{i}\right)$ is identically zero on $V_{1}$ for $1 \leq i \leq k$. This implies that $\Phi\left(V_{1}\right) \subseteq V_{2}$. By the maximum principle, $\Phi\left(X_{1}\right) \cap \Phi\left(V_{1}-\bar{X}_{1}\right)=\emptyset$. It follows that $\Phi^{-1}\left(X_{2}\right)=X_{1}$ and $\Phi$ is a proper map from $V_{1}$ to $V_{2}$. By the proper mapping theorem, $\Phi\left(V_{1}\right)$ is a complex variety.

We claim that $\operatorname{dim} \Phi\left(V_{1}\right)=n$. If $\operatorname{dim} \Phi\left(V_{1}\right)<n$, then for some $q$ in $\Phi\left(V_{1}\right), \Phi^{-1}(q)$ is a compact variety of dimension at least one sitting inside $V_{1}$. This gives a contradiction since $V_{1}$ is Stein. As $\Phi\left(V_{1}\right) \subseteq V_{2}$ and $\operatorname{dim} \Phi\left(V_{1}\right)=n=\operatorname{dim} V_{2}$, we have $\Phi\left(V_{1}\right)=V_{2}$. It follows that $\Phi\left(X_{1}\right)=X_{2}$. A local computation of J. E. Fornæss ([Fo, Proposition 12]) would apply to show that $\Phi$ is a local biholomorphism near $X_{1}$. (This was observed independently by Pinchuk [Pi].) In particular, $\Phi: V_{1}-\Phi^{-1}\left(S_{2} \cup \Phi\left(S_{1}\right)\right) \rightarrow V_{2}-\left(S_{2} \cup\right.$ $\left.\Phi\left(S_{1}\right)\right)$ is locally biholomorphic and hence is a finite covering.

Let $p \in S_{1}$ and $q=\Phi(p)$. We claim $q \in S_{2}$. Suppose on the contrary that $q$ is a smooth point in $V_{2}$; then $\Phi$ maps a neighborhood $U_{1}$ of $p$ to a neighborhood $U_{2}$ of $q$ as a branch covering. Since $p$ is a normal singularity, the punctured neighborhood $U_{1}-p$ of $p$ is connected. On the other hand, the punctured neighborhood $U_{2}-\{q\}$ of $q$ is simply connected because $q$ is a smooth point. We conclude that $\left.\Phi\right|_{U_{1}}: U_{1} \rightarrow U_{2}$ is one-to-one and onto. By the Hartogs extension theorem, the inverse map $\left.\Phi^{-1}\right|_{U_{2}-\{q\}}: U_{2}-\{q\} \rightarrow$ $U_{1}-\{p\}$ can be holomorphically extended onto $U_{2}$. It follows that $\left.\Phi\right|_{U_{1}}: U_{1} \rightarrow U_{2}$ is a biholomorphic map. This leads to a contradiction. Therefore $\Phi\left(S_{1}\right) \subseteq S_{2}$ and $\Phi$ : $V_{1}-\Phi^{-1}\left(S_{2}\right) \rightarrow V_{2}-S_{2}$ is a covering map.

Now assume that $S_{2}$ does not have a quotient singularity. Let $q$ be any point in $S_{2}$. We need to show that $\Phi^{-1}(q) \subseteq S_{1}$. If $\Phi^{-1}(q)$ is not contained in $S_{1}$, then there exists a smooth point $q^{\prime}$ of $V_{1}$ in $\Phi^{-1}(q)$. Recall that $\Phi^{-1}(q)$ is a finite set. We can find an open neighborhood $U$ of $q^{\prime}$ which is biholomorphic to a domain in $\mathbb{C}^{n}$ such that $\left.\Phi\right|_{U}$ from $U$ to the germ of $\left(V_{2}, q\right)$ is a branch covering with ramification locus $\left\{q^{\prime}\right\}$. By Theorem 1 of [ $[\mathrm{Pr}]$, we conclude that $\left(V_{2}, q\right)$ is a quotient singularity. This is a contradiction.

Proof of Corollary 1.1. It follows immediately from the proof of Proposition 1.1

Proof of Proposition 1.2. By Proposition 1.1, if there exists a non-constant CR morphism $\phi: X_{1} \rightarrow X_{2}$, then $\Phi$ can be extended as a ramified covering map from $V_{1}$ to $V_{2}$ with ramification locus $S_{2}$. Since $V_{1}$ is smooth, by the proof of Proposition $1.1, S_{2}$ consists of only quotient singularities and hence the geometric genus of these singularities is zero. It follows that $p_{g}\left(X_{2}\right)=0$ in view of Remark 2.1(b). This leads to a contradiction.

Proof of Proposition 1.3 Let $V_{i}$ be a normal variety in $\mathbb{C}^{N_{i}}$ with only isolated singularities such that $\partial V_{i}=X_{i}, i=1,2$. Let $S_{1}$ and $S_{2}$ be the singular set of $V_{1}$ and $V_{2}$ respectively. Let $\Phi: X_{1} \rightarrow X_{2}$ be a non-constant CR morphism. In view of Proposition 1.1. $\Phi$ can be extended to a proper surjective holomorphic map from $V_{1}$ to $V_{2}$ such that $\Phi\left(S_{1}\right)=S_{2}$, and $\Phi: V_{1}-\Phi^{-1}\left(S_{2}\right) \rightarrow V_{2}-S_{2}$ is a covering map. There is a natural map

$$
\Phi^{*}: \frac{\Gamma\left(V_{2}-S_{2}, \Omega^{n}\right)}{L^{2}\left(V_{2}-S_{2}, \Omega^{n}\right)} \rightarrow \frac{\Gamma\left(V_{1}-\Phi^{-1}\left(S_{2}\right), \Omega^{n}\right)}{L^{2}\left(V_{1}-\Phi^{-1}\left(S_{2}\right), \Omega^{n}\right)} .
$$


Since $\Phi: V_{1}-\Phi^{-1}\left(S_{2}\right) \rightarrow V_{2}-S_{2}$ is a finite covering map, a form $\omega \in \Gamma\left(V_{2}-S_{2}, \Omega^{n}\right)$ is $L^{2}$-integrable if and only if $\Phi^{*}(\omega)$ is $L^{2}$-integrable. Thus $\Phi^{*}$ is injective. Observe that $\Phi^{-1}\left(S_{2}\right)-S_{1}$ is a discrete subset in the smooth part of $V_{1}$. By Hartogs' theorem, $\Gamma\left(V_{1}-\Phi^{-1}\left(S_{2}\right), \Omega^{n}\right)=\Gamma\left(V_{1}-S_{1}, \Omega^{n}\right)$ and $L^{2}\left(V_{1}-\Phi^{-1}\left(S_{2}\right), \Omega^{n}\right)=L^{2}\left(V_{1}-S_{1}, \Omega^{n}\right)$. It follows that $p_{g}\left(X_{2}\right) \leq p_{g}\left(X_{1}\right)$.

Proof of Theorem 1.1. Let $\Phi: X_{1} \rightarrow X_{2}$ be a non-constant CR morphism. Proposition 1.1 says that $\Phi$ can be extended to a proper surjective holomorphic map from $V_{1}$ to $V_{2}$ such that $\Phi\left(S_{1}\right) \subseteq S_{2}$ and $\Phi: V_{1}-\Phi^{-1}\left(S_{2}\right) \rightarrow V_{2}-S_{2}$ is a covering map of degree $d$. For any $q \in S_{2}$, we know that the punctured neighborhood of $q$ in $V_{2}$ is $\left(2 n-N_{2}-1\right)$ connected in view of a theorem of Hamm [Ha]. Since $2 n-N_{2}-1 \geq 1$ by assumption, the punctured neighborhood of $q$ is simply connected.

We claim that $\Phi^{-1}(q) \subseteq S_{1}$. If $\Phi^{-1}(q)$ is not contained in $S_{1}$, then there exists a smooth point $q^{\prime}$ of $V_{1}$ in $\Phi^{-1}(q)$. Recall that $\Phi^{-1}(q)$ is a finite set. We can find an open neighborhood $U$ of $q^{\prime}$ which is biholomorphic to a domain in $\mathbb{C}^{n}$ such that $\left.\Phi\right|_{U}$ from $U$ to the germ of $\left(V_{2}, q\right)$ is a branch covering with ramification locus $\left\{q^{\prime}\right\}$. Since the punctured neighborhood of $q$ in $V_{2}$ is simply connected, this implies $\left.\Phi\right|_{U}$ is injective and hence $\left.\Phi\right|_{U}$ is a biholomorphism. This leads to a contradiction because $q$ is a singular point.

We have shown that $\Phi^{-1}(q)=\left\{q_{1}^{\prime}, \ldots, q_{d}^{\prime}\right\} \subseteq S_{1}$. Since $\left|S_{1}\right| \leq 2\left|S_{2}\right|-1$, by the pigeon-hole principle, there exists $q \in S_{2}$ such that $\Phi^{-1}(q)=\left\{q_{1}^{\prime}\right\}$. Since the punctured neighborhood of $q$ is simply connected, we conclude that the degree of the covering map $\Phi: V_{1}-S_{1} \rightarrow V_{2}-S_{2}$ is one.

Proof of Theorem 1.2 Let $\Phi: X \rightarrow X$ be a non-constant CR morphism. In view of Proposition 1.1 and the fact that the singular set $S=\left\{q_{1}, \ldots, q_{m}\right\}$ of $V$ does not contain any quotient singularity, $\Phi$ can be extended to a proper holomorphic map from $V$ to $V$ such that $\Phi^{-1}(S)=S$ and $\Phi: V-S \rightarrow V-S$ is a covering map of degree $d$. Let $\pi: M \rightarrow V$ be the minimal good resolution of $V$ such that the exceptional sets $E_{1}=$ $\pi^{-1}\left(q_{1}\right)=\bigcup_{i=1}^{\ell_{1}} A_{i}^{1}, \ldots, E_{m}=\pi^{-1}\left(q_{m}\right)=\bigcup_{i=1}^{\ell_{m}} A_{i}^{m}$ are normal crossing divisors. Consider the fiber product $V \underset{V}{\times} M$ of the maps $\Phi: V \rightarrow V$ and $\pi: M \rightarrow V$. Let $\tau: \tilde{M} \rightarrow V \underset{V}{\times} M$ be the normalization map. Then we have the following commutative diagram where $\pi_{1}$ and $\pi_{2}$ are natural projections:

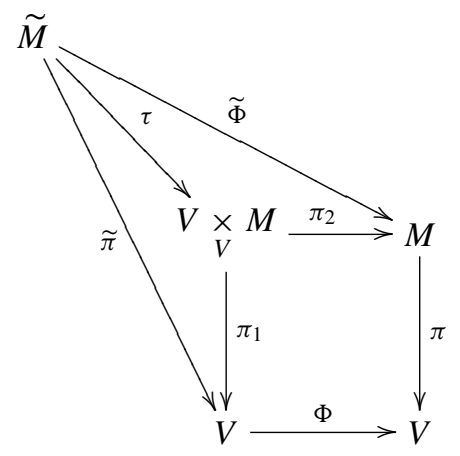


Notice that $\pi_{1}: \underset{V}{V} \underset{V}{\times} M \rightarrow V$ is a biholomorphism outside $\pi_{1}^{-1}(S)$, and $\pi_{2}: V \underset{V}{V} M \rightarrow M$ is a covering map outside $\bigcup_{i=1}^{m} E_{i}$. Thus $\widetilde{\Phi}:=\pi_{2} \circ \tau: \widetilde{M} \rightarrow M$ is a $d$-fold branch covering. For each $A_{i}^{j} \subseteq E_{j}$, and any point $q_{i}^{j} \in A_{i}^{j}$ which is smooth in $\bigcup_{i=1}^{m} E_{i}$, we choose a germ of a curve $\Gamma_{i}^{j}$ at the point $q_{i}^{j}$ which intersects $\bigcup_{i=1}^{m} E_{i}$ only at $q_{i}^{j}$ and the intersection of $A_{i}^{j}$ and $\Gamma_{i}^{j}$ is transversal at $q_{i}^{j}$. Let $\Gamma=\bigcup\left\{\Gamma_{i}^{j}: 1 \leq j \leq m, 1 \leq i \leq \ell_{j}\right\}$. Notice that $\tilde{\pi}:=\pi_{1} \circ \tau$ is a finite proper map which is a biholomorphism outside $\widetilde{E}:=$ $\tilde{\pi}^{-1}(S)=\widetilde{\Phi}^{-1}(E)$ where $E=E_{1} \cup \cdots \cup E_{m}$. Observe that $\widetilde{E}$ has exactly $m$ connected components, $\widetilde{E}=\widetilde{E}_{1} \cup \cdots \cup \widetilde{E}_{m}$. Clearly $\widetilde{\Phi}_{*}(\widetilde{E})=\sum_{i, j} d_{i}^{j} A_{i}^{j}$ where $d_{i}^{j} \leq d$.

By the projection formula (cf. p. 34 of [Fu], or p. 426 of [Har])

$$
\sum_{j=1}^{m} \sum_{i=1}^{\ell_{j}} d_{i}^{j}=\Gamma \cdot \widetilde{\Phi}_{*}(\widetilde{E})=\widetilde{\Phi}^{*}(\Gamma) \cdot \widetilde{E} \geq\left(\ell_{1}+\cdots+\ell_{m}\right) d
$$

The last inequality comes from the fact that $\Phi^{*}\left(\Gamma_{i}^{j}\right)$ has $d$ distinct branches because $\Phi: V-S \rightarrow V-S$ is a $d$-fold covering map. Since $d_{i}^{j} \leq d$, we conclude that $d_{i}^{j}=d$ for all $i, j$. It follows that the branch locus of $\widetilde{\Phi}$ is contained in the singular locus of $\bigcup_{i=1}^{m} E_{i}$, which is of dimension zero. As $\widetilde{M}$ is normal and $M$ is smooth, $\widetilde{\Phi}: \widetilde{M} \rightarrow M$ is a covering map by purity of branch locus. Hence $\widetilde{M}$ is smooth and $\tilde{\pi}: \widetilde{M} \rightarrow V$ is also a resolution of singularity. Thus if $\Phi\left(q_{i}\right)=q_{j}$, then $\left(V, q_{i}\right)$ and $\left(V, q_{j}\right)$ are isomorphic as germs of singularities. This is because each resolution of $\left(V, q_{j}\right)$ is a resolution of $\left(V, q_{i}\right)$. Observe that $\pi_{1}^{-1}(S)$ as a reduced analytic set is isomorphic to $\pi^{-1}(S)$ via $\pi_{2}$. The number of irreducible components of $\tilde{\pi}^{-1}(S)$ is at least the same those of $\pi^{-1}(S)$.

Let $A_{1}$ be an irreducible component of $E$ and $\widetilde{A}_{1}$ be an irreducible component of $\widetilde{\Phi}^{-1}\left(A_{1}\right)$. Then $\left.\widetilde{\Phi}\right|_{\widetilde{A}_{1}}: \widetilde{A}_{1} \rightarrow A_{1}$ is a covering map. Suppose that the degree of $\left.\widetilde{\Phi}\right|_{\widetilde{A}_{1}}$ is $d_{1}$. Observe that by the projection formula we have

$$
\begin{aligned}
\widetilde{A}_{1} \cdot \widetilde{A}_{1} & =\left(\left.\widetilde{\Phi}\right|_{\widetilde{A}_{1}} ^{*} A_{1}\right)\left(\left.\widetilde{\Phi}\right|_{\widetilde{A}_{1}} ^{*} A_{1}\right)=A_{1} \cdot\left(\left.\left.\widetilde{\Phi}\right|_{\widetilde{A}_{1 *}} \widetilde{\Phi}\right|_{A_{1}} ^{*} A_{1}\right)=\left.A_{1} \cdot \widetilde{\Phi}\right|_{\widetilde{A}_{1 *}} \widetilde{A}_{1}=A_{1} \cdot\left(d_{1} A_{1}\right) \\
& =d_{1}\left(A_{1} \cdot A_{1}\right) .
\end{aligned}
$$

Suppose that $M$ is actually a minimal resolution of $V$, i.e. there is no rational curve with self-intersection number -1 . By the Riemann-Hurwitz formula (see p. 218 of [Gr-Ha] $), g\left(\widetilde{A}_{1}\right)>0$ if $g\left(A_{1}\right)>0$. In view of (3.1), $\widetilde{M}$ has no rational curve with self-intersection number -1 . Thus $\tilde{M}$ is also a minimal resolution of $V$. It follows from the uniqueness of minimal resolution that the degree of $\Phi$ is one.

If $M$ is not a minimal resolution of $V$, then there exists a rational curve $A_{1}$ with selfintersection number -1 . Let $\widetilde{A}_{1}$ be an irreducible component of $\widetilde{\Phi}^{-1}\left(A_{1}\right)$. Then $\widetilde{A}_{1}$ is smooth and $\left.\widetilde{\Phi}\right|_{\widetilde{A}_{1}}: \widetilde{A}_{1} \rightarrow A_{1}$ is a covering map. Let $d_{1}$ be the degree of $\left.\widetilde{\Phi}\right|_{\tilde{A}_{1}}$. Then $\chi_{T}\left(\widetilde{A}_{1}\right)=d_{1} \chi_{T}\left(A_{1}\right)$, where $\chi_{T}$ is the topological Euler characteristic. Since the only curves with positive topological Euler characteristic are rational curves and their topological Euler characteristic must be 2 , it follows that $\widetilde{A}_{1}$ is a rational curve and $d_{1}=1$. In view of (3.1), $\widetilde{A}_{1} \cdot \widetilde{A}_{1}=-1$. Because $\widetilde{\Phi}$ is a $d$-fold covering, $\widetilde{\Phi}^{-1}\left(A_{1}\right)$ has exactly $d$ connected components $\widetilde{A}_{11}, \widetilde{A}_{12}, \ldots, \widetilde{A}_{1 d}$, which are all rational curves with self-intersection -1 . 
By blowing down $\widetilde{A}_{11}, \widetilde{A}_{12}, \ldots, \widetilde{A}_{1 d}$ in $\widetilde{M}$ and $A_{1}$ in $M$, we obtain $\widetilde{M}_{1}$ and $M_{1}$ respectively. The map $\widetilde{\Phi}_{1}: \widetilde{M}_{1} \rightarrow M_{1}$ induced by $\widetilde{\Phi}$ is still a $d$-fold covering. After a finite number of steps, we get $M_{k}$ and $\widetilde{M}_{k}$ where $\widetilde{M}_{k}$ is the minimal resolution of $V$. Since the map $\widetilde{\Phi}_{k}: \widetilde{M}_{k} \rightarrow M_{k}$ is still a $d$-fold covering, by our previous argument above, we conclude that $d=1$.

Proof of Proposition 1.4 Let $\Phi: X \rightarrow X$ be a non-constant CR morphism. By Proposition 1.1. $\Phi$ can be extended to a holomorphic covering map from $V$ to $V$. Now we follow Pinchuk's argument in [Pi]. $\Phi$ induces a homomorphism of the fundamental groups $\Phi_{*}: \pi_{1}(V) \rightarrow \pi_{1}(V)$. It is clear that $\Phi$ is biholomorphic if and only if $\Phi_{*}$ is an isomorphism. Clearly $\Phi_{*}$ is a monomorphism. We shall examine the iteration $\Phi^{k}=\Phi \circ \Phi^{k-1}$. By the Montel Theorem, there exists a subsequence $\Phi^{k_{i}}(i=1,2, \ldots)$ which converges to $\Psi: V \rightarrow V$ uniformly on compact sets. Since $V$ is a complex manifold with smooth boundary, its fundamental group has a finite number of generators $\omega_{1}, \ldots, \omega_{\ell}$. On each curve $\omega_{\ell}, \Phi^{k_{i}}$ converges to $\Psi$ uniformly. Therefore for $p$ sufficiently large, we have $\Phi_{*}^{k_{p}}=\Psi_{*}$. Hence $\Psi_{*}$ is a monomorphism. Now observe that

$$
\Psi_{*}=\Phi_{*}^{k_{p+1}}=\Phi_{*}^{k_{p}} \circ \Phi_{*}^{k_{p+1}-k_{p}}=\Psi_{*} \circ \Phi_{*}^{k_{p+1}-k_{p}} .
$$

We conclude that $\Phi_{*}^{k_{p+1}-k_{p}}$ and consequently $\Phi_{*}$ is an epimorphism.

Acknowledgments. We thank L. Ein, P. Griffith, H. Hironaka, X. Huang and A. Libgober for some stimulating discussions. We also thank the referee who provided many useful comments to improve the presentation of our paper. In particular it was pointed out that the conclusion of our Theorem 1.3 is wrong if the singular set is empty: the map $(z, w) \mapsto\left(z, w^{2}\right)$ from $\mathbb{C} \times \mathbb{C}^{*}$ to itself provides a counter-example.

This research was partially supported by NSF.

\section{References}

[Al] Alexander, H.: Proper holomorphic mappings in $\mathbb{C}^{n}$. Indiana Univ. Math. J. 26, 137-146 (1977) Zbl 0391.32015 MR 0422699

[An-Gr] Andreotti, A., Grauert, H.: Théorèmes de finitude pour la cohomologie des espaces complexes. Bull. Soc. Math. France 90, 193-259 (1962) Zbl 0106.05501 MR 0150342

[Be-Ca] Bell, S., Catlin, D.: Boundary regularity of proper holomorphic mappings. Duke Math. J. 49, 385-396 (1982) Zbl 0475.32011 MR 0659947

[BM] Boutet de Monvel, L.: Intégration des équations de Cauchy-Riemann induites formelles. In: Séminaire Goulaouic-Lions-Schwartz, 1974-1975, exp. IX, 14 pp. (1975) Zbl 0317.58003 MR 0409893

[Di-Fo] Diederich, K., Fornæss, J.: Boundary regularity of proper holomorphic mappings. Invent. Math. 67, 363-384 (1982) Zbl 0501.32010 MR 0664111

[Fo] Fornæss, J.: Embedding strictly pseudoconvex domains in convex domains. Amer. J. Math. 98, 529-569 (1976) Zbl 0334.32020 MR 0422683

[Fu] Fulton, W.: Intersection Theory. 2nd ed., Springer (1998) Zbl 0885.14002 MR 1644323 
[Gr-Ha] Griffiths, P., Harris, J.: Principles of Algebraic Geometry. Wiley (1978) Zbl 0408.14001 MR 0507725

[Ha] Hamm, H.: On the vanishing of local homotopy groups for isolated singularities of complex spaces. J. Reine Angew. Math. 323, 172-176 (1981) Zbl 0483.32007 MR 0611450

[Ha-La1] Harvey, F. R., Lawson, H. B.: On boundaries of complex analytic varieties I. Ann. of Math. 102, 223-290 (1975) Zbl 0317.32017 MR 0425173

[Ha-La2] Harvey, F. R., Lawson, H. B.: Addendum to Theorem 10.4 in: Boundaries of Analytic Varieties, arXiv:math/0002195v1[math.CV] (2000)

[Har] Hartshorne, R.: Algebraic Geometry. Grad. Texts in Math. 52, Springer (1977) Zbl 0367.14001 MR 0463157

[Hi] Hironaka, H.: Resolution of singularities of an algebraic variety over a field of characteristic 0, I-II. Ann. of Math. 79, 109-326 (1964) Zbl 0122.38603 MR 0199184

[Ko] Kohn, J.: Boundaries of complex manifolds. In: Proc. Conf. Complex Analysis (Minneapolis, MN, 1964), Springer, Berlin, 81-94 (1965) Zbl 0166.36003 MR 0175149

[Ko-Oc] Kobayashi, S., Ochiai, T.: Meromorphic mappings onto compact spaces of general type, Invent. Math. 31, 7-16 (1975) Zbl 0331.32020 MR 0402127

[La] Laufer, H.: Normal Two-Dimensional Singularities. Ann. of Math. Stud. 71, Princeton Univ. Press, 1971. Zbl 0245.32005 MR 0320365

[Lu-Ya] Luk, H.-S., Yau, S. S.-T.: Kohn-Rossi cohomology and its application to the complex Plateau problem II. J. Differential Geom. 77, 135-148 (2007) Zbl 1123.32020 MR 2344356

[Mo] Mostow, G. D.: Strong Rigidity of Locally Symmetric Space. Ann. of Math. Stud. 78, Princeton University Press, (1973) Zbl 0265.53039 MR 0385004

[Pi] Pinchuk, S.: On proper holomorphic mappings of strictly pseudoconvex domains. Siberian Math. J. 15, 644-649 (1974) Zbl 0303.32016 MR 0355109

[Pr] Prill, D.: Local classification of quotients of complex manifolds by discontinuous groups. Duke Math. J. 34, 375-386 (1967) Zbl 0179.12301 MR 0210944

[Si] Siu, Y.-T.: The complex-analyticity of harmonic maps and the strong rigidity of compact Kähler manifolds. Ann. of Math. (2) 112, 73-111 (1980) Zbl 0517.53058 MR 0584075

[St] Steenbrink, J.: Mixed Hodge structure on the vanishing cohomology. In: Real and Complex Singularities (Oslo, 1976), C. P. Holm (ed.), Sijthoff and Noordhoff, 525-563 (1977) Zbl 0373.14007 MR 0485870

[Ta] Tanaka, N.: A Differential Geometric Study on Strongly Pseudoconvex Manifolds. Lectures in Math. Kyoto Univ. 9, Kinokuniya (1975) Zbl 0331.53025 MR 0399517

[Ya1] Yau, S. S.-T.: Two theorems on higher dimensional singularities. Math. Ann. 231, 55-59 (1977) Zbl 0343.32010 MR 0492389

[Ya2] Yau, S. S.-T.: Global invariants for strongly pseudoconvex varieties with isolated singularities: Bergman functions. Math. Res. Lett. 11, 809-832 (2004) Zbl 1076.32024 MR 2106243

[Ya-Yu] Yau, S. S.-T., Yu, Y.: Algebraic classification of rational CR structures on topological 5sphere with transversal holomorphic $S^{1}$-action in $\mathbb{C}^{4}$. Math. Nachr. 246-247, 207-233 (2002) Zbl 1019.32021 MR 1944558 